\title{
Comparison of Smartphone Pedometer Apps on a Treadmill versus
} Outdoors

IVÁN A. FIGUEROA, JESÚS P. GONZÁLEZ, PERLA LEYVA, JOSÉ L. GÁMEZ, NAOMI LUCIO, ELVIA V. SALAZAR, CINDY SALAZAR, MIRIAM GARCIA, and MERRILL FUNK.

Exercise Science Lab; Department of Health and Human Performance; University of Texas Rio Grande Valley; Brownsville TX University of Texas School of Public Health-Health Science Center at Houston Brownsville Regional Campus

Category: Master

Advisor / Mentor: Funk, Merrill (Merrill.funk@utrgv.edu)

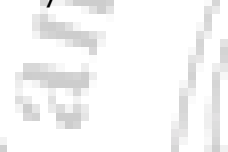

\section{ABSTRACT}

Previous research has focused on the accuracy of smartphone pedometer apps in laboratory settings, however less information is available in outdoor (free living) environments. PURPOSE: Determine the accuracy of 5 smartphone apps at recording steps at a walking speed in a laboratory versus an outdoor setting. METHODS: Twenty-three healthy college students consented (Mean \pm SD; 22 $\pm 3.8 \mathrm{yrs}$; BMI $24.9 \pm 4.13 \mathrm{~kg} / \mathrm{m}^{2}$ ) to participate in 2 separate visits. During the first visit participants walked 500 steps at $3 \mathrm{mph}$ on a treadmill while wearing a pedometer and a smartphone placed in the pocket using 5 pedometer apps concurrently (Moves, Google Fit (G-Fit), Runtastic, Accupedo, S-Health). During the second visit, participants walked 400 meters at $3 \mathrm{mph}$ on a sidewalk outside. Actual steps for each visit were recorded using a hand tally counter device. Zero and negative values were replaced with the mean value for that trial. Statistical analyses were performed using IBM SPSS 23.0. Mean bias scores were calculated between the step count for each app and the respective tally count for each trial. Mean bias scores were correlated between trials for each app using Pearson correlations and significance was set at $\mathrm{p}<0.05$. Mean Absolute Percent Error (MAPE) values were also calculated for each app for both trials. RESULTS: G-Fit recorded 2 zero values and 2 negative values and Moves recorded 1 zero value. Mean bias scores were significantly correlated between the indoor and outdoor protocols for the pedometer $(\mathrm{r}=0.67, \mathrm{p}<0.01)$ and S-Health $(\mathrm{r}=0.46, \mathrm{p}<0.5)$. The remaining apps were not correlated between protocols. The outdoor protocol producing a greater mean bias for the outdoor protocol for G-Fit, Runtastic, and Accupedo (mean bias \pm SD indoor, outdoor; $-4.3 \pm 53.1,-19.3 \pm 120.0 ;-10.7 \pm 63.3,-33.4 \pm 118.7 ; 16.0 \pm 143.6$, 79.0 \pm 75.0 ; respectively) and a greater mean bias for the indoor protocol for the pedometer, Moves, and SHealth (mean bias indoor, outdoor; $-1.4 \pm 41.5,0.0 \pm 34.1 ;-117.4 \pm 196.7,-42.2 \pm 209.6$; $11.3 \pm 28.4,0.0 \pm 58.7$; respectively). MAPE was below $5 \%$ for the pedometer and S-Health for both trials. CONCLUSION: Apps with the lowest error in a controlled setting may be less affected when used in other settings, while apps with greater variation in a controlled setting may be affected when used in a different environment. 